\title{
STATISTICAL STABILITY FOR MULTIDIMENSIONAL PIECEWISE EXPANDING MAPS
}

\author{
JOSÉ F. ALVES, ANTONIO PUMARIÑO, AND ENRIQUE VIGIL
}

\begin{abstract}
We present sufficient conditions for the (strong) statistical stability of some classes of multidimensional piecewise expanding maps. As a consequence we get that a certain natural two-dimensional extension of the classical one-dimensional family of tent maps is statistically stable.
\end{abstract}

\section{Contents}

1. Introduction

1.1. Statistical stability

1.2. Piecewise expanding maps

1.3. Two-dimensional tent maps

2. Functions of bounded variation

3. Piecewise expanding maps

4. Two-dimensional tent maps

References

\section{INTRODUCTION}

Along this paper we deal with multidimensional piecewise expanding maps defined in some compact subset of an Euclidean space. A first approach to this topic has been made in dimension one by Lasota and Yorke in [12, where they proved the existence of absolutely continuous invariant probability measures for a class of piecewise $C^{2}$ expanding maps of the interval. The extension to higher dimensions in general is a very delicate question, mostly because of the intricate geometry of the domains of smoothness and their images under iterations. In the last decades many results have appeared in the literature with several different approaches. A first result in dimension two was obtained by Keller in [11]. In the multidimensional case, Gora and Boyarski in [9] proved the existence of absolutely continuous invariant probability measures for maps with a finite number of domains of smoothness under some condition of no cusps in the domains of smoothness. This result

Date: August 29, 2018.

2010 Mathematics Subject Classification. 37A05, 37A10, 37C75.

Key words and phrases. Piecewise expanding maps, Physical measures, Statistical stability.

JFA was partially funded by Fundação Calouste Gulbenkian, by the European Regional Development Fund through the program COMPETE and by the Portuguese Government through FCT under the projects PEst-C/MAT/UI0144/2013 and PTDC/MAT/120346/2010. AP and EV were partially supported by MEC grant MTM2011-22956, and the Foundation for the promotion in Asturias of the Scientific and Technologic Research BP12-123. 
was later extended by Adl-Zarabi in [1] to piecewise expanding maps allowing cusps in the domains of smoothness, and by Alves in [2] to piecewise expanding maps with countably many domains of smoothness. Similar results were drawn in the particular case of piecewise linear maps by Buzzi and Tsujii in [5, 20, and by the same authors in the case of piecewise real analytic expanding maps of the plane in [6, 19]. A general result on the existence of absolutely continuous invariant probability measures in any finite dimension was given by Saussol in [17] for $C^{1+}$ piecewise expanding maps with infinitely many domains of smoothness under some control on the accumulation of discontinuities under iterations of the map.

Many concrete situations lead to the appearance of families of piecewise expanding maps under conditions that guarantee the existence of absolutely continuous invariant probability measures, in many cases this probability measure being unique. It is then a natural question trying to decide weather these measures depend continuously on the dynamics, i.e the statistical stability of those maps. This question was addressed in [4] for certain robust classes of maps with non-uniform expansion; see also [3]. In [13], the authors consider a one-parameter family $\left(\Lambda_{t}\right)_{t}$ of two-dimensional piecewise linear maps defined on a triangle in $\mathbb{R}^{2}$. This family $\left(\Lambda_{t}\right)_{t}$ is closely related to the family of limit return maps arising when certain three-dimensional homoclinic bifurcations take place; see [18. It is showed in [13, Proposition 6.1] and the comments following it that $\Lambda_{t}$ becomes the best choice in the piecewise linear setting for describing the dynamics of the original limit return maps given in [18] when the unstable manifold of the saddle point has dimension two.

The results in the present paper have a twofold aim: to give sufficient conditions for the statistical stability of some general classes of multidimensional piecewise expanding maps; and to prove the statistical stability of the family of maps introduced in [13]. This last result will be obtained as an application of our general result.

1.1. Statistical stability. In this work we consider discrete-time dynamical systems defined in a compact region $R \subset \mathbb{R}^{d}$, for some $d \geq 1$. Given a measurable map $\phi: R \rightarrow R$, we say that a probability measure $\mu$ on the Borel sets of $R$ is $\phi$-invariant if $\mu\left(\phi^{-1}(A)\right)=\mu(A)$, for any Borel set $A \subset R$. If $\mu(A)=0$ whenever $m(A)=0$, where $m$ denotes the Lebesgue measure on the Borel sets of $\mathbb{R}^{d}$, then $\mu$ is called absolutely continuous. In this case, there exists an $m$-integrable function $h \geq 0$, usually denoted $d \mu / d m$ and called the density of $\mu$ with respect to $m$, such that for any Borel set $A \subset R$ we have $\mu(A)=\int_{A} h d m$. A $\phi$ invariant probability measure $\mu$ is called ergodic if $\mu(A) \mu(R \backslash A)=0$, whenever $\phi^{-1}(A)=A$. As a consequence of Birkhoff's Ergodic Theorem we have that any $\phi$-invariant absolutely continuous ergodic probability measure $\mu$ is a physical measure, meaning that for a subset of points $x \in R$ with positive Lebesgue measure we have

$$
\lim _{n \rightarrow \infty} \sum_{j=0}^{n-1} f\left(\phi^{j}(x)\right)=\int f d \mu
$$

for all continuous $f: R \rightarrow \mathbb{R}$.

Let $I$ be a metric space and $\left(\phi_{t}\right)_{t \in I}$ a family of maps $\phi_{t}: R \rightarrow R$ such that each $\phi_{t}$ has some absolutely continuous $\phi_{t}$-invariant probability measure. We say that the family $\left(\phi_{t}\right)_{t \in I}$ is statistically stable if for any $t_{0} \in I$, any choice of a sequence $\left(t_{n}\right)_{n}$ in $I$ converging to $t_{0}$ and any choice of a sequence of absolutely continuous $\phi_{t_{n}}$-invariant probability measures $\left(\mu_{t_{n}}\right)_{n}$, then any accumulation point of the sequence of densities 
$d \mu_{t_{n}} / d m$ must converge in the $L^{1}$-norm to the density of an absolutely continuous $\phi_{t_{0}}{ }^{-}$ invariant probability measure. Of course, when each $\phi_{t}$ has a unique absolutely continuous invariant probability measure $\mu_{t}$, then statistical stability means that $d \mu_{t} / d m$ converges in the $L^{1}$-norm to $d \mu_{t_{0}} / d m$ when $t \rightarrow t_{0}$. A strictly weaker notion of statistical stability may be given if we assume only weak* convergence of the measures $\mu_{t}$ to $\mu_{t_{0}}$ when $t \rightarrow t_{0}$.

1.2. Piecewise expanding maps. Here we state precisely sufficient conditions for the statistical stability of certain higher dimensional families of $C^{2}$ piecewise expanding maps with countably many domains of smoothness. We follow the approach in [2] which, on its turn, was inspired in [9].

Let $R$ be a compact set in $\mathbb{R}^{d}$, for some $d \geq 1$. For each $1 \leq p \leq \infty$ we denote by $L^{p}(R)$ the Banach space of functions in $L^{p}(m)$ with support contained in $R$, endowed with the usual norm $\|\quad\|_{p}$. Let $\phi: R \rightarrow R$ a map for which there is a (Lebesgue mod 0) partition $\left\{R_{i}\right\}_{i=1}^{\infty}$ of $R$ such that each $R_{i}$ is a closed domain with piecewise $C^{2}$ boundary of finite $(d-1)$-dimensional measure and $\phi_{i}=\phi \mid R_{i}$ is a $C^{2}$ bijection from $\operatorname{int}\left(R_{i}\right)$, the interior of $R_{i}$, onto its image with a $C^{2}$ extension to $R_{i}$. We say that $\phi$ is piecewise expanding if

$\left(\mathrm{P}_{1}\right)$ there is $0<\sigma<1$ such that for every $i \geq 1$ and $x \in \operatorname{int}\left(\phi\left(R_{i}\right)\right)$

$$
\left\|D \phi_{i}^{-1}(x)\right\|<\sigma .
$$

We say that $\phi$ has bounded distortion if

$\left(\mathrm{P}_{2}\right)$ there is $D \geq 0$ such that for every $i \geq 1$ and $x \in \operatorname{int}\left(\phi\left(R_{i}\right)\right)$

$$
\frac{\left\|D\left(J \circ \phi_{i}^{-1}\right)(x)\right\|}{\left|J \circ \phi_{i}^{-1}(x)\right|} \leq D,
$$

where $J$ is the Jacobian of $\phi$.

Finally, we say that $\phi$ has long branches if

$\left(\mathrm{P}_{3}\right)$ there are $\beta, \rho>0$ and for each $i \geq 1$ there is a $C^{1}$ unitary vector field $X_{i}$ in $\partial \phi\left(R_{i}\right)$ such that:

(a) the segments joining each $x \in \partial \phi\left(R_{i}\right)$ to $x+\rho X_{i}(x)$ are pairwise disjoint and contained in $\phi\left(R_{i}\right)$, and their union forms a neighborhood of $\partial \phi\left(R_{i}\right)$ in $\phi\left(R_{i}\right)$.

(b) for every $x \in \partial \phi\left(R_{i}\right)$ and $v \in T_{x} \partial \phi\left(R_{i}\right) \backslash\{0\}$ the angle $\angle\left(v, X_{i}(x)\right)$ between $v$ and $X_{i}(x)$ satisfies $\left|\sin \angle\left(v, X_{i}(x)\right)\right| \geq \beta$.

Here we assume that at the singular points $x \in \partial \phi\left(R_{i}\right)$ where $\partial \phi\left(R_{i}\right)$ is not smooth the vector $X_{i}(x)$ is a common $C^{1}$ extension of $X_{i}$ restricted to each $(d-1)$-dimensional smooth component of $\partial \phi\left(R_{i}\right)$ having $x$ in its boundary. We also assume that the tangent space of any such singular point $x$ is the union of the tangent spaces to the $(d-1)$-dimensional smooth components it belongs to.

In the one-dimensional case $d=1$, condition $\left(\mathrm{P}_{3}\right)(\mathrm{a})$ is clearly satisfied once we take the sets in the partition of $R$ as being intervals whose images $\phi\left(R_{i}\right)$ have sizes uniformly bounded away from zero. Additionally, condition $\left(\mathrm{P}_{3}\right)($ a) always holds in dimension one, since $\phi\left(R_{i}\right)$ is a 0 -dimensional manifold and so $T_{x} \partial \phi\left(R_{i}\right)=\{0\}$ for any $x \in \phi\left(R_{i}\right)$. In this case we can even take the optimal value $\beta=1$; see Remark 3.2.

Theorem A. Let I be a metric space and $\left(\phi_{t}\right)_{t \in I}$ a family of $C^{2}$ piecewise expanding maps $\phi_{t}: R \rightarrow R$ with bounded distortion and long branches. Assume that for each $t \in I$

(1) for each continuous $f: R \rightarrow \mathbb{R}$ we have $\left\|f \circ \phi_{t^{\prime}}-f \circ \phi_{t}\right\|_{d} \rightarrow 0$ when $t^{\prime} \rightarrow t$; 
(2) there exist $0<\lambda<1$ and $K>0$ for which

$$
\sigma_{t}\left(1+\frac{1}{\beta_{t}}\right) \leq \lambda \quad \text { and } \quad D_{t}+\frac{1}{\beta_{t} \rho_{t}}+\frac{D_{t}}{\beta_{t}} \leq K
$$

where $\sigma_{t}, D_{t}, \beta_{t}, \rho_{t}$ are constants for which $\left(P_{1}\right),\left(P_{2}\right)$ and $\left(P_{3}\right)$ hold for $\phi_{t}$.

Then $\left(\phi_{t}\right)_{t \in I}$ is statistically stable.

It follows from [2, Section 5] that under the assumptions above each $\phi_{t}$ has a finite number of ergodic absolutely continuous invariant probability measures. The proof of this result uses the space of functions of bounded variation in $\mathbb{R}^{d}$, which are known to belong to the space $L^{p}(R)$, with $p=d /(d-1)$; see (3) below. Observing that $1 / p+1 / d=1$, this makes the choice of the norm \|\|$_{d}$ in condition (1) less mysterious; see the proof of Lemma 3.5. Notice that condition (1) in Theorem A holds whenever the maps $\phi_{t}$ are continuous and $\phi_{t}$ depends continuously (in the $C^{0}$-norm) on $t \in I$.

1.3. Two-dimensional tent maps. Here we present the family of maps introduced in [13] and give some result on its statistical stability. We define the family of maps $\Lambda_{t}: T \rightarrow T$ on the triangle $T=T_{0} \cup T_{1}$, where

$$
T_{0}=\{(x, y): 0 \leq x \leq 1,0 \leq y \leq x\}, \quad T_{1}=\{(x, y): 1 \leq x \leq 2,0 \leq y \leq 2-x\},
$$

and

$$
\Lambda_{t}(x, y)= \begin{cases}(t(x+y), t(x-y)), & \text { if }(x, y) \in T_{0} \\ (t(2-x+y), t(2-x-y)), & \text { if }(x, y) \in T_{1}\end{cases}
$$

The domains $T_{0}$ and $T_{1}$ are separated by a straight line segment $\mathcal{C}=\left\{\left(x_{1}, x_{2}\right) \in T: x_{1}=1\right\}$ that we call the critical set of $\Lambda_{t}$.

As shown in [15], the map $\Lambda_{1}$ displays the same properties of the one-dimensional tent map $\lambda_{2}(x)=1-2|x|$. Among them, the consecutive pre-images $\left\{\Lambda_{1}^{-n}(\mathcal{C})\right\}_{n \in \mathbb{N}}$ of the critical line $\mathcal{C}$ define a sequence of partitions (whose diameter tends to zero as $n$ goes to infinity) of $T$ leading them to conjugate $\Lambda_{1}$ to a one sided shift with two symbols. Hence, it easily follows that $\Lambda_{1}$ is transitive in $T$. Furthermore, for every point $\left(x_{0}, y_{0}\right) \in T$ whose orbit never hits the critical line the Lyapounov exponent of $\Lambda_{1}$ along the orbit of $\left(x_{0}, y_{0}\right)$ is positive (and coincides with $\frac{1}{2} \log 2$ ) in all nonzero direction. Finally, it can be constructed an absolutely continuous ergodic invariant probability measure for $\Lambda_{1}$; see [15]. Because of this, $\Lambda_{1}$ was called the two-dimensional tent map. Since the parameter $t$ in (2) essentially gives the rate of expansion for $\Lambda_{t}$ (playing the same roll of the parameter $a$ for $\lambda_{a}(x)=1-a|x|$ ), the family $\left(\Lambda_{t}\right)_{t}$ can be considered as a natural extension of the one-dimensional family of tent maps and naturally called a family of two-dimensional tent maps.

The results obtained in [15] for $t=1$ were extended to a larger set of parameters. More precisely, it was proved in [14] that for each $t \in[\tau, 1]$, with $\tau=\frac{1}{\sqrt{2}}(\sqrt{2}+1)^{\frac{1}{4}} \approx 0.882$, the map $\Lambda_{t}$ exhibits a strange attractor $A_{t} \subset T: \Lambda_{t}$ is (strongly) transitive in $A_{t}$, the periodic orbits are dense in $A_{t}$, and there exists a dense orbit in $A_{t}$ with two positive Lyapunov exponents. Furthermore, $A_{t}$ supports a unique absolutely continuous $\Lambda_{t}$-invariant ergodic probability measure $\mu_{t}$. As an application of Theorem A we shall obtain the following result.

Theorem B. The family $\left(\Lambda_{t}\right)_{t \in[\tau, 1]}$ is statistically stable. 
As each $\Lambda_{t}$ has a unique absolutely continuous invariant probability measure $\mu_{t}$, the statistical stability means in this case that $d \mu_{t} / d m$ converges in the $L^{1}$-norm to $d \mu_{t_{0}} / d m$ when $t \rightarrow t_{0}$, for each $t_{0} \in[\tau, 1]$.

\section{FunCtions OF BOUNDED VARIATION}

The main ingredient for the proof of Theorem $\mathrm{A}$ is the notion of variation for functions in multidimensional spaces. We adopt the definition given in [8]. Given $f \in L^{1}\left(\mathbb{R}^{d}\right)$ with compact support we define the variation of $f$ as

$$
V(f)=\sup \left\{\int_{\mathbb{R}^{n}} f \operatorname{div}(g) d m: g \in C_{0}^{1}\left(\mathbb{R}^{d}, \mathbb{R}^{d}\right) \text { and }\|g\| \leq 1\right\},
$$

where $C_{0}^{1}\left(\mathbb{R}^{d}, \mathbb{R}^{d}\right)$ is the set of $C^{1}$ functions from $\mathbb{R}^{d}$ to $\mathbb{R}^{d}$ with compact support, $\operatorname{div}(g)$ is the divergence of $g$ and \|\| is the sup norm in $C_{0}^{1}\left(\mathbb{R}^{d}, \mathbb{R}^{d}\right)$. Given a bounded set $R \subset \mathbb{R}^{d}$ we consider the space of bounded variation functions in $L^{1}(R)$

$$
B V(R)=\left\{f \in L^{1}(R): V(f)<+\infty\right\} .
$$

Contrarily to the classical one-dimensional definition of bounded variation, a multidimensional bounded variation function need not to be bounded; see [10. However, by Sobolev's Inequality (see e.g. [ 8 , Theorem 1.28]) there is some constant $C>0$ (only depending on the dimension $d$ ) such that for any $f \in B V(R)$

$$
\left(\int|f|^{p} d m_{d}\right)^{1 / p} \leq C V(f), \quad \text { with } \quad p=\frac{d}{d-1} .
$$

This in particular gives $B V(R) \subset L^{p}(R)$. We shall use the following properties of bounded variation functions whose proofs may be found in [7] or [8]:

$\left(\mathrm{B}_{1}\right) B V(R)$ is dense in $L^{1}(R)$;

$\left(\mathrm{B}_{2}\right)$ if $\left(f_{k}\right)_{k}$ is a sequence in $B V(R)$ converging to $f$ in the $L^{1}$-norm, then $V(f) \leq$ $\liminf _{k} V\left(f_{k}\right)$;

$\left(\mathrm{B}_{3}\right)$ if $\left(f_{k}\right)_{k}$ is a sequence in $B V(R)$ such that $\left(\left\|f_{k}\right\|_{1}\right)_{k}$ and $\left(V\left(f_{k}\right)\right)_{k}$ are bounded, then $\left(f_{k}\right)_{k}$ has some subsequence converging in the $L^{1}$-norm to a function in $B V(R)$.

\section{PiECEWISE EXPANDING MAPS}

In this section we prove Theorem $\mathrm{A}$. Let $\left\{R_{i}^{t}\right\}_{i=1}^{\infty}$ be the domains of smoothness of $\phi_{t}$ with $t \in I$ satisfying the assumptions of Theorem $\mathrm{\AA}$ and define $\phi_{t, i}=\left.\phi_{t}\right|_{R_{i}^{t}}$ for all $i \geq 1$. For each $t \in I$ we consider the Perron-Frobenius operator

$$
P_{t}: L^{1}(R) \longrightarrow L^{1}(R)
$$

defined for $f \in L^{1}(R)$ as

$$
P_{t} f=\sum_{i=1}^{\infty} \frac{f \circ \phi_{t, i}^{-1}}{\left|J \circ \phi_{t, i}^{-1}\right|} \chi_{\phi_{t}\left(R_{i}^{t}\right)} .
$$

It is well known that the following two properties hold for each $P_{t}$.

$\left(\mathrm{C}_{1}\right)\left\|P_{t} f\right\|_{1} \leq\|f\|_{1}$ for every $f \in L^{1}(R)$;

$\left(\mathrm{C}_{2}\right) P_{t} f=f$ if and only if $f$ is the density of an absolutely continuous $\phi_{t}$-invariant probability measure. 
Considering $0<\lambda<1$ and $K>0$ as in the statement of Theorem $\mathrm{A}$, the proof of the next lemma follows immediately from [2, Lemma $5.4 \&$ Lemma 5.5] with

$$
K_{1}=K \sum_{j=0}^{\infty} \lambda^{j} .
$$

Lemma 3.1. Given $t \in I$ and $j \geq 1$ we have for each $f \in B V(R)$

$$
V\left(P_{t}^{j} f\right) \leq \lambda^{j} V(f)+K_{1}\|f\|_{1} .
$$

Remark 3.2. The proof of [2, Lemma 5.4] uses [9, Lemma 3] applied to the sets $S=\phi\left(R_{i}\right)$, which gives for a function $f \in C^{1}(S)$,

$$
\int_{\partial S}|f| d m \leq \frac{1}{\beta}\left(\frac{1}{\rho} \int_{S}|f| d m+\int_{S}\|D f\| d m\right) .
$$

In the one-dimensional case we have for any interval $S$ and $x \in S$

$$
f(x) \leq \frac{1}{|S|} \int_{S}|f| d m+\int_{S}|D f| d m,
$$

which yields a formula similar to (4) in the one-dimensional case with $\beta=1$.

In the proof of the result below we follow some standard arguments with functions of bounded variation, namely those used in [12] for the one-dimensional case.

Proposition 3.3. Given $t \in I$ and $f \in L^{1}(R)$ the sequence $1 / n \sum_{j=0}^{n-1} P_{t}^{j} f$ has some accumulation point in the $L^{1}$-norm. Moreover, any such accumulation point belongs to $B V(R)$ and has variation bounded by $4 K_{1}\|f\|_{1}$.

Proof. Given $f \in L^{1}(R)$, by property $\left(\mathrm{B}_{1}\right)$ we may consider a sequence of functions $\left(f_{k}\right)_{k}$ in $B V(R)$ converging to $f$ in the $L^{1}$-norm. With no loss of generality we may assume that $\left\|f_{k}\right\|_{1} \leq 2\|f\|_{1}$ for every $k \geq 1$. It follows from Lemma 3.1 that for each $k \geq 1$ and large $j$ we have

$$
V\left(P_{t}^{j} f_{k}\right) \leq \lambda^{j} V\left(f_{k}\right)+K_{1}\left\|f_{k}\right\|_{1} \leq 3 K_{1}\|f\|_{1} .
$$

So, for large $n$ we have

$$
V\left(\frac{1}{n} \sum_{j=0}^{n-1} P_{t}^{j} f_{k}\right) \leq 4 K_{1}\|f\|_{1} .
$$

Using that $\left\|f_{k}\right\|_{1} \leq 2\|f\|_{1}$ for every $k \geq 1$, it easily follows from $\left(\mathrm{C}_{1}\right)$ that

$$
\left\|\frac{1}{n} \sum_{j=0}^{n-1} P_{t}^{j} f_{k}\right\|_{1} \leq 2\|f\|_{1} .
$$

Then it follows from $\left(\mathrm{B}_{3}\right)$ that there exists some $g_{k} \in B V(R)$ and a sequence $\left(n_{i}\right)_{i}$ such that $1 / n_{i} \sum_{j=0}^{n_{i}-1} P_{t}^{j} f_{k}$ converges in the $L^{1}$-norm to $g_{k}$ as $i$ goes to $+\infty$. Moreover, by $\left(\mathrm{B}_{2}\right)$ we have $V\left(g_{k}\right) \leq 4 K_{1}\|f\|_{1}$ for every $k \geq 1$. Hence, we may apply the same argument to the sequence $\left(g_{k}\right)_{k}$ and obtain a subsequence $\left(k_{i}\right)_{i}$ such that $\left(g_{k_{i}}\right)_{i}$ converges in the $L^{1}$-norm to some $g \in B V(R)$ with $V(g) \leq 4 K_{1}\|f\|_{1}$. Hence, there must be some sequence $\left(n_{\ell}\right)_{\ell}$ 
converging to $+\infty$ for which $1 / n_{\ell} \sum_{j=0}^{n_{\ell}-1} P_{t}^{j} f_{k_{\ell}}$ converges to $g$ in the $L^{1}$-norm as $\ell \rightarrow+\infty$. On the other hand,

$$
\left\|\frac{1}{n_{\ell}} \sum_{j=0}^{n_{\ell}-1}\left(P_{t}^{j} f_{k_{\ell}}-P_{t}^{j} f\right)\right\|_{1} \leq \frac{1}{n_{\ell}} \sum_{j=0}^{n_{\ell}-1}\left\|f_{k_{\ell}}-f\right\|_{1}=\left\|f_{k_{\ell}}-f\right\|_{1}
$$

and this last term goes to 0 as $\ell \rightarrow+\infty$. This clearly gives that $1 / n_{\ell} \sum_{j=0}^{n_{\ell}-1} P_{t}^{j} f$ converges to $g$ in the $L^{1}$-norm.

To prove the second part of the lemma, consider some subsequence of $1 / n \sum_{j=0}^{n-1} P_{t}^{j} f$ converging to $f_{0}$ in the $L^{1}$-norm. Taking that subsequence playing the role of the whole sequence in the argument above we easily see that $f_{0}$ satisfies the conclusion by uniqueness of the limit.

Corollary 3.4. If $h_{t}$ is the density of an absolutely continuous $\phi_{t}$-invariant probability measure, then $h_{t} \in B V(R)$ and $V\left(h_{t}\right) \leq 4 K_{1}$.

Proof. Take $h_{t}$ the density of an absolutely continuous $\phi_{t}$-invariant probability measure. We have from property $\left(\mathrm{C}_{2}\right)$ that $P_{t}^{j} h_{t}=h_{t}$ for all $j \geq 1$. This implies that the sequence $1 / n \sum_{j=0}^{n-1} P_{t}^{j} h_{t}$ is constant and equal to $h_{t}$, and so the result follows.

Now we are in conditions to conclude the proof of Theorem A,

Let $\left(t_{n}\right)_{n}$ be a sequence in $I$ converging to some $t_{0} \in I$. Assume that for each $n \geq 1$ we have an absolutely continuous $\phi_{t_{n}}$-invariant probability measure $\mu_{n}$ and consider

$$
h_{n}=\frac{d \mu_{n}}{d m} .
$$

Using the fact that each $h_{n}$ is the density of a probability measure and Corollary 3.4 we have for all $n \geq 1$

$$
\left\|h_{n}\right\|_{1}=1 \quad \text { and } \quad V\left(h_{n}\right) \leq 4 K_{1} .
$$

Hence, by $\left(\mathrm{B}_{2}\right)$ and $\left(\mathrm{B}_{3}\right)$ there exists $h_{0} \in B V$ with $V\left(h_{0}\right) \leq 4 K_{1}$ such that the sequence $\left(h_{n}\right)_{n}$ converges to $h_{0}$ in the $L^{1}$-norm. Let $\mu_{0}$ be the probability measure in $R$ whose density with respect to $m$ is $h_{0}$. Theorem $\mathrm{A}$ is now a consequence of the following lemma.

Lemma 3.5. $\mu_{0}$ is a $\phi_{t_{0}}$-invariant measure.

Proof. Since the sequence $\left(h_{n}\right)_{n}$ converges to $h_{0}$ in the $L^{1}$-norm, it easily follows that $\left(\mu_{n}\right)_{n}$ converges to $\mu_{0}$ in the weak* topology. Thus, given any $f: R \rightarrow \mathbb{R}$ continuous we have

$$
\int f d \mu_{n} \rightarrow \int f d \mu, \text { when } n \rightarrow \infty .
$$

On the other hand, since $\mu_{n}$ is $\phi_{t_{n}}$-invariant we have

$$
\int f d \mu_{n}=\int\left(f \circ \phi_{t_{n}}\right) d \mu_{n}, \text { for every } n \text {. }
$$

It is enough to prove that

$$
\int\left(f \circ \phi_{t_{n}}\right) d \mu_{n} \longrightarrow \int\left(f \circ \phi_{t_{0}}\right) d \mu, \quad \text { when } n \rightarrow \infty .
$$


We have

$$
\begin{aligned}
\left|\int\left(f \circ \phi_{t_{n}}\right) d \mu_{n}-\int\left(f \circ \phi_{t_{0}}\right) d \mu\right| \\
\leq\left|\int\left(f \circ \phi_{t_{n}}\right) d \mu_{n}-\int\left(f \circ \phi_{t_{0}}\right) d \mu_{n}\right|+\left|\int\left(f \circ \phi_{t_{0}}\right) d \mu_{n}-\int\left(f \circ \phi_{t_{0}}\right) d \mu\right| \\
\leq \int\left|f \circ \phi_{t_{n}}-f \circ \phi_{t_{0}}\right| d \mu_{n}+\left|\int\left(f \circ \phi_{t_{0}}\right) d \mu_{n}-\int\left(f \circ \phi_{t_{0}}\right) d \mu\right| \\
\quad=\int\left|f \circ \phi_{t_{n}}-f \circ \phi_{t_{0}}\right| h_{n} d m+\left|\int\left(f \circ \phi_{t_{0}}\right)\left(h_{n}-h_{0}\right) d m\right| .
\end{aligned}
$$

Now using (3) we easily get that each $h_{n} \in L^{p}(R)$ with $p=d /(d-1)$ and

$$
\left\|h_{n}\right\|_{p} \leq C V\left(h_{n}\right) \leq 4 C K_{1} .
$$

Observing that $1 / p+1 / d=1$, then by Hölder's Inequality we get

$$
\int\left|f \circ \phi_{t_{n}}-f \circ \phi_{t_{0}}\right| h_{n} d m \leq\left\|f \circ \phi_{t_{n}}-f \circ \phi_{t_{0}}\right\|_{d} \cdot\left\|h_{n}\right\|_{p} \leq 4 C_{d} K_{1}\left\|f \circ \phi_{t_{n}}-f \circ \phi_{t_{0}}\right\|_{d}
$$

and this clearly converges to zero, when $n \rightarrow+\infty$, by assumption (1) in the statement of Theorem A. On the other hand, as $f$ is bounded we have

$$
\left|\int\left(f \circ \phi_{t_{0}}\right)\left(h_{n}-h_{0}\right) d m\right| \leq\left\|f \circ \phi_{t_{0}}\right\|_{\infty} \cdot\left\|h_{n}-h_{0}\right\|_{1}
$$

and this clearly converges to 0 when $n \rightarrow+\infty$ as well.

\section{TWO-Dimensional TEnT MAPS}

In this section we shall prove Theorem B. The idea is to obtain it as a corollary of Theorem A. As observed before, each $\Lambda_{t}$ is strongly transitive: any open set becomes the whole space under a finite number of iterations by $\Lambda_{t}$. This implies that the absolutely continuous $\Lambda_{t}$-invariant ergodic probability measure $\mu_{t}$ must be unique. Moreover, any power of $\Lambda_{t}$ has a unique absolutely continuous invariant ergodic probability measure as well, which must necessarily coincide with $\mu_{t}$. Thus, it is enough to obtain the statistical stability for some power of the maps in our family.

We are going to see that the family $\left(\Lambda_{t}^{3}\right)_{t \in[\tau, 1]}$ is in the conditions of Theorem A. Namely, each $\Lambda_{t}^{3}: T \rightarrow T$ is a $C^{2}$ piecewise expanding map with bounded distortion and long branches with constants $\sigma_{t}, D_{t}, \beta_{t}, \rho_{t}$ satisfying $\left(\mathrm{P}_{1}\right),\left(\mathrm{P}_{2}\right)$ and $\left(\mathrm{P}_{3}\right)$, and

$$
\sigma_{t}\left(1+\frac{1}{\beta_{t}}\right) \leq \lambda \text { and } D_{t}+\frac{1}{\beta_{t} \rho_{t}}+\frac{D_{t}}{\beta_{t}} \leq K,
$$

for some choice of uniform constants $0<\lambda<1$ and $K>0$; observe that as the maps $\Lambda_{t}^{3}$ are continuous then the first condition in Theorem $\mathrm{A}$ is trivially satisfied.

From the definition of $\Lambda_{t}$ in (11) and (2) we obviously have that $T_{0}$ and $T_{1}$ are the domains of smoothness of $\Lambda_{t}$. The map $\Lambda_{t}$ is piecewise linear with

$$
D \Lambda_{t}(x)=\left(\begin{array}{cc}
t & t \\
t & -t
\end{array}\right)
$$


for $x \in T_{0} \backslash \mathcal{C}$, and

$$
D \Lambda_{t}(x)=\left(\begin{array}{cc}
-t & t \\
-t & -t
\end{array}\right)
$$

for $x \in T_{1} \backslash \mathcal{C}$. From here we deduce that for all $x \in T \backslash \mathcal{C}$ we have

$$
\left\|D \Lambda_{t}^{-1}(x)\right\| \leq \frac{1}{2 t}
$$

Now take $R=T$ and $\left\{R_{i}^{t}\right\}_{i=1}^{8}$ the (Lebesgue mod 0) partition of $R$ given by the domains of smoothness of $\Lambda_{t}^{3}$. From (6) we easily deduce that

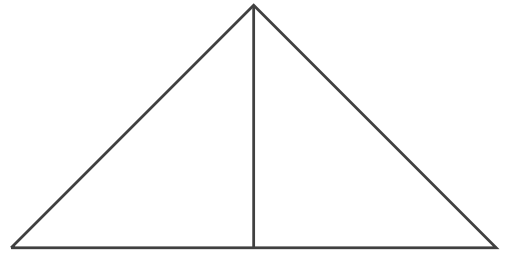

(a)

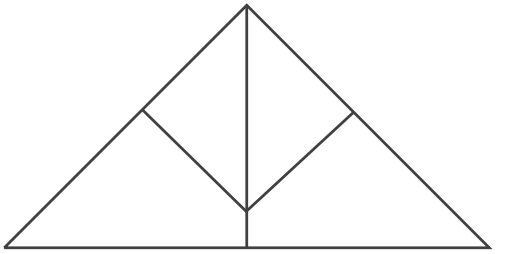

(b)

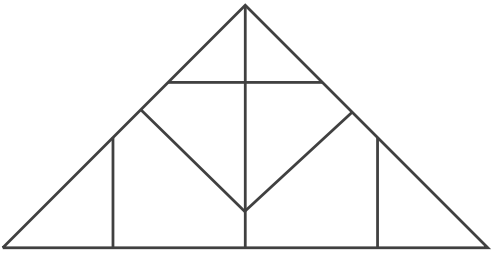

(c)

Figure 1. Smoothness domains: (a) for $\Lambda_{t} \quad$ (b) for $\Lambda_{t}^{2} \quad$ (c) for $\Lambda_{t}^{3}$

$$
\left\|\left(D \Lambda_{t}^{3}\right)^{-1}(x)\right\| \leq \frac{1}{8 t^{3}}:=\sigma_{t}<1
$$

and so property $\left(\mathrm{P}_{1}\right)$ holds for each $t \in[\tau, 1]$; recall that $\tau \approx 0.88$. Since $\Lambda_{t}^{3}$ is linear on each domain of smoothness, then it has 0 distortion. Thus we obtain property $\left(\mathrm{P}_{2}\right)$ with $D_{t}=0$, for each $t \in[\tau, 1]$.

Let us now check $\left(\mathrm{P}_{3}\right)$. As each $\Lambda_{t}^{3}$ is linear on each $R_{i}^{t}$ and preserves angles, it is enough to obtain the geometric property $\left(\mathrm{P}_{3}\right)$ for the domains $R_{i}^{t}$ 's instead of their images. Since the pre-image of the critical set $\mathcal{C}$ delimits the boundary of the domains of smoothness, it easily follows that the boundary of each $R_{i}^{t}$ is formed by at most five straight line segments with slope $-1,0,1$ or $\infty$ meeting at an angle at least $\pi / 4$; see Figure 1(c). Then, it is

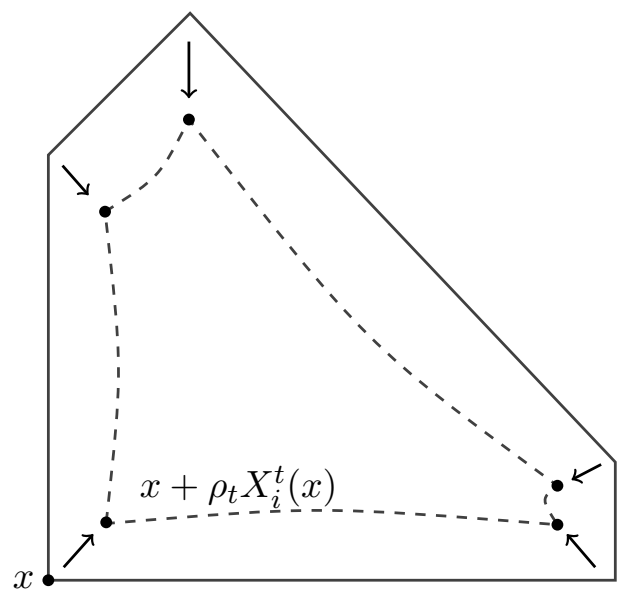

Figure 2. A long branch for $\Lambda_{t}^{3}$ 
not hard to check that for every $t \in[\tau, 1]$ and $i=1, \ldots, 8$ there is a piecewise $C^{1}$ unitary vector filed $X_{i}^{t}$ in $\partial R_{i}^{t}$ such that

$$
\left|\sin \angle\left(v, X_{i}^{t}(x)\right)\right| \geq \sin \frac{\pi}{8}:=\beta_{t}
$$

for every $x \in \partial R_{i}^{t}$ and $v \in T_{x} \partial R_{i}^{t} \backslash\{0\}$; see Figure 2, To prove the existence of $\rho_{t}$ is is enough to observe that the domains of smoothness of $\Lambda_{t}^{3}$ depend continuously on the parameter $t$ as illustrated in Figure 3, and so it is possible to choose an uniform value of $\rho$ such that $\left(\mathrm{P}_{3}\right)$ holds for each $t \in[\tau, 1]$.

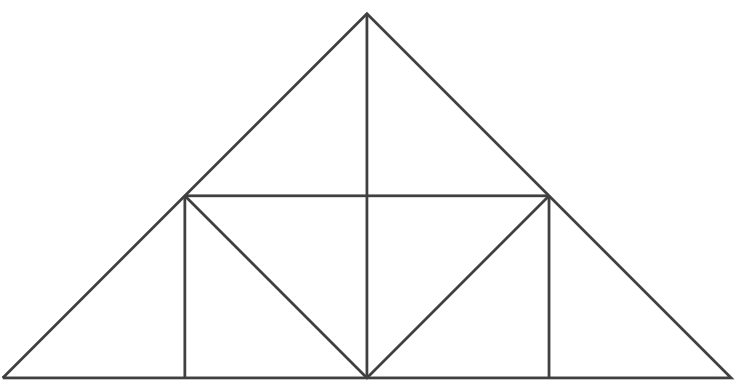

(a)

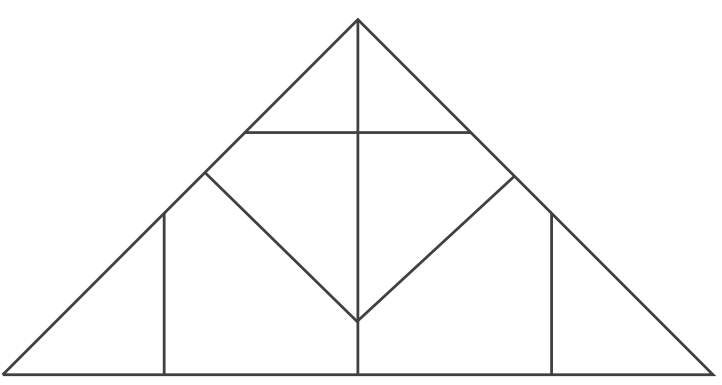

(b)

Figure 3. Domains of smoothness: (a) for $\Lambda_{1}^{3} \quad$ (b) for $\Lambda_{\tau}^{3}$

Altogether this shows that there are $0<\lambda<1$ and $K>0$ such that

$$
\sigma_{t}\left(1+\frac{1}{\beta_{t}}\right) \leq \frac{1}{8 t^{3}}\left(1+\frac{1}{\sin (\pi / 8)}\right) \leq \lambda
$$

for every $t \in[\tau, 1]$ (recall that $\tau \approx 0.88$ ) and

$$
D_{t}+\frac{1}{\beta_{t} \rho_{t}}+\frac{D_{t}}{\beta_{t}}=\frac{1}{\rho \sin (\pi / 8)}:=K,
$$

thus having proved (5) and hence Theorem B.

\section{REFERENCES}

[1] K. Adl-Zarabi. Absolutely continuous invariant measures for piecewise expanding $C^{2}$ transformations in $\mathbf{R}^{n}$ on domains with cusps on the boundaries. Ergodic Theory Dynam. Systems, 16(1):1-18, 1996.

[2] J. F. Alves. SRB measures for non-hyperbolic systems with multidimensional expansion. Ann. Sci. École Norm. Sup. (4), 33(1):1-32, 2000.

[3] J. F. Alves. Strong statistical stability of non-uniformly expanding maps. Nonlinearity, 17(4):1193$1215,2004$.

[4] J. F. Alves and M. Viana. Statistical stability for robust classes of maps with non-uniform expansion. Ergodic Theory Dynam. Systems, 22(1):1-32, 2002.

[5] J. Buzzi. Absolutely continuous invariant measures for generic multi-dimensional piecewise affine expanding maps. Internat. J. Bifur. Chaos Appl. Sci. Engrg., 9(9):1743-1750, 1999. Discrete dynamical systems.

[6] J. Buzzi. Absolutely continuous invariant probability measures for arbitrary expanding piecewise $\mathbf{R}$ analytic mappings of the plane. Ergodic Theory Dynam. Systems, 20(3):697-708, 2000.

[7] L. C. Evans and R. F. Gariepy. Measure theory and fine properties of functions. Studies in Advanced Mathematics. CRC Press, Boca Raton, FL, 1992.

[8] E. Giusti. Minimal surfaces and functions of bounded variation, volume 80 of Monographs in Mathematics. Birkhäuser Verlag, Basel, 1984. 
[9] P. Góra and A. Boyarsky. Absolutely continuous invariant measures for piecewise expanding $C^{2}$ transformation in $\mathbf{R}^{N}$. Israel J. Math., 67(3):272-286, 1989.

[10] P. Góra and A. Boyarsky. On functions of bounded variation in higher dimensions. Amer. Math. Monthly, 99(2):159-160, 1992.

[11] G. Keller. Ergodicité et mesures invariantes pour les transformations dilatantes par morceaux d'une région bornée du plan. C. R. Acad. Sci. Paris Sér. A-B, 289(12):A625-A627, 1979.

[12] A. Lasota and J. A. Yorke. On the existence of invariant measures for piecewise monotonic transformations. Trans. Amer. Math. Soc., 186:481-488, 1973.

[13] A. Pumariño, J. A. Rodríguez, J. C. Tatjer, and E. Vigil. Expanding Baker maps as models for the dynamics emerging from 3D-homoclinic bifurcations. Discrete Contin. Dyn. Syst. Ser. B, 19(2):523$541,2014$.

[14] A. Pumariño, J. A. Rodríguez, J. C. Tatjer, and E. Vigil. Chaotic dynamics for 2-d tent maps, 2014. http://www.ma.utexas.edu/mp_arc/c/14/14-8.pdf

[15] A. Pumariño and J. C. Tatjer. Dynamics near homoclinic bifurcations of three-dimensional dissipative diffeomorphisms. Nonlinearity, 19(12):2833-2852, 2006.

[16] A. Pumariño and J. C. Tatjer. Attractors for return maps near homoclinic tangencies of threedimensional dissipative diffeomorphisms. Discrete Contin. Dyn. Syst. Ser. B, 8(4):971-1005, 2007.

[17] B. Saussol. Absolutely continuous invariant measures for multidimensional expanding maps. Israel $J$. Math., 116:223-248, 2000.

[18] J. C. Tatjer. Three-dimensional dissipative diffeomorphisms with homoclinic tangencies. Ergodic Theory Dynam. Systems, 21(1):249-302, 2001.

[19] M. Tsujii. Absolutely continuous invariant measures for piecewise real-analytic expanding maps on the plane. Comm. Math. Phys., 208(3):605-622, 2000.

[20] M. Tsujii. Absolutely continuous invariant measures for expanding piecewise linear maps. Invent. Math., 143(2):349-373, 2001.

José F. Alves, Centro de Matemática da Universidade do Porto, Rua do Campo Alegre 687, 4169-007 Porto, Portugal

E-mail address: jfalves@fc.up.pt

$U R L:$ http://www.fc.up.pt/cmup/jfalves

Antonio Pumariño, Departamento de Matemáticas, Facultad de Ciencias de la UniverSidad de Oviedo, Calvo Sotelo s/n, 33007 Oviedo, Spain.

E-mail address: apv@uniovi.es

Enrique Vigil, Departamento de Matemáticas, Facultad de Ciencias de la Universidad de Oviedo, Calvo Sotelo s/n, 33007 Oviedo, Spain.

E-mail address: vigilkike@gmail.com 